\title{
Project portfolio optimisation and scheduling using temporal tolerances
}

\author{
G. Calbert $^{\mathrm{a}}$ \\ ${ }^{a}$ Defence Science and Technology Group, Edinburgh, Adelaide, South Australia, 5111 \\ Email: Greg.Calbert@dst.defence.gov.au
}

\begin{abstract}
In financial portfolio optimisation, there is a hitherto agreed definition of the value of a particular series of investments, this being financial returns. When one considers applying the machinery of portfolio optimisation in Defence, the objective is to select and schedule a subgroup of projects. Such projects are drawn from a larger pool, typically extant and new projects. There is no fiscal "return," as projects are public goods. Each project must be assigned a value depending on its potential start time. Ideally each project has two attributes, a vector of starting-time dependent values and a vector of costs which commence from the project starting time. The optimised portfolio ensemble must meet time-dependent budget constraints.

The optimisation formalism is that of a multiple knapsack problem and the methods of integer programming apply. In practice forming starting-time project values is contentious. Unlike financial portfolio theory, there is no agreed approach to value determination, as there is no analogy to currency. It is this issue that drives the approach developed here. The starting-time values for projects are elicited through a specific pre-defined time-dependent functional form of value. The parameters of this functional form are then derived by asking what is the ideal project starting-time and how tolerant is the subject matter expert of that time? By doing this, one introduces an agreed currency for projects, deviance from this ideal time. This pre-defined functional form of the starting-time values, is called in this paper a temporal tolerance function.

In this paper the multi-knapsack problem is explored with temporal tolerance starting-time value functions. In Section 1 the issue of determining the value of the public good's military capability is discussed. In Section 2 then describe the multiple-knapsack problem and introduce a number of temporal tolerance functions. In Section 3 computational experiments are conducted with such functions to determine portfolio structure properties, such as optimised value and time deviance as tolerance levels change. Finally in Section 4 the paper is summarised and the general problem understanding the statistics of project selection, as a function of cost structure and value function structure is discussed.
\end{abstract}

Keywords: Portfolio, optimisation, public goods, value 


\section{INTRODUCTION}

Financial institutions such as banks or superannuation funds often seek to invest in projects or organisations, that under some definition maximise different metrics of return whilst moderating risk. At the heart of such decisions is a hitherto agreed to measure of return, that of money or a probability distribution of potential monetary returns. Money is a community-wide measure of value. While there is some controversy with different nations' comparative currency values, as is evidenced in price purchasing parity-dollar discrepancies, such differences are well understood.

In the parlance of economics, goods and services are valued according to two criteria Lumen (2021, (accessed 6 July, 2021)). These criteria are excludability and rivality. Excludable goods are possessed by agents only if they can pay for them. Rival goods are those where the possession by one agent prevents the use by another.

Defence capabilities are public goods. These capabilities, by definition of the term public good are both nonexcludable and non-rivalrous. Individuals can benefit from defence capabilities even if they do not pay for it and multiple individuals (such as a nation state) can benefit from defence capabilities simultaneously.

Because defence capabilities are public goods, it is difficult to define a single numerical measure of value either to the capability as a whole or to the projects that constitute its entirety. The problem of valuing public goods also occurs in the settings of health care policy and that of environmental protection. Surveys may be conducted to measure levels of "happiness" or "satisfaction" Levinson (2009, (accessed 15 July 2021)).

Yet a clear definition of value for a defence capability is precisely what is required if one is to apply the techniques of mathematical optimisation to solve the defence portfolio problem. Here, the multiple knapsack problem applies Kellerer, Hans and Pferschy, Ulrich and Pisinger, David (2004). For a general review on the application of the knapsack problem to defence portfolio optimisation see Harrison et al. (2020).

There are a number of approaches to the elicitation of value of a military capabilities. One can conduct a survey amongst subject matter experts (SMEs) and seek an average. One can also calculate, through war-gaming the probability that some objective is met given the capability is present, compared with that when the capability is absent. If $\Omega(t)$ is the required objective state given the project starts at time $t$, and $i$ is the capability then the value $V_{i t}$ could be estimated as $\operatorname{Pr}(\Omega(t) \mid i)-\operatorname{Pr}(\Omega(t) \mid \neg i)$. There are several issues with this approach. First, while such probabilities could be estimated through the use of conditional decomposition in a directed or undirected graphical model, the number of parameters required to form such a model is generally large and the computations NP-hard Heckman, D (2001).

Rather that focusing on some arbitrary form of project value, one may impose a pre-defined functional form across time. By imposing a specific starting-time value functional form, project timing itself can become the main subject of questions to SMEs. Instead of asking what is the value of a project at some time $t$ ? the question what is the ideal scheduled time you wish the project to start and how tolerant are you of that time? is asked. This approach is taken for two reasons. One, the concept of time is well understood and is not an abstracted concept such as public goods value. Two, in the context of defence portfolios, military SMEs will generally have an understanding of the starting times of potential foreign military projects and or technologies with varying degrees of accuracy. Thus in the portfolio selection process asking an SME an ideal starting time directly infers a time to counter some possible adversarial capability.

Portfolio planners can at the outset select a time window through which projects can start. Suppose a set of projects is indexed by $P=\{1,2, \ldots,|P|\}$. If $x_{i t}$ is the decision variable to commence project $i \in P$ at time $t \in\{1,2, \ldots, T\}$ and $\left[\tau, \tau^{\prime}\right]$ is the starting-time window, one could impose the constraint that $\sum_{t<\tau} x_{i t}+$ $\sum_{t>\tau^{\prime}}^{T} x_{i t}=0$. This constraint-based approach could also be implemented by appropriate selection of the starting-time values $V_{i t}$. For example if one sets $V_{i t} \leq 0$ for $t \in[0, \tau-1]$ and $t \in\left[\tau^{\prime}, T\right]$ there is no value increase in choosing starting times outside the window $\left[\tau, \tau^{\prime}\right]$. Thus one could define for $v>0$,

$$
V_{i t}=\left\{\begin{array}{l}
v \text { if } t \in\left[\tau, \tau^{\prime}\right] \\
0 \text { otherwise }
\end{array}\right.
$$

as a simple functional form for starting-time values to specify the time window that project $i$ can commence. In turn, this thinking may be extended to consider other functional forms for $V_{i t}$. In the next section 2, we consider other functional forms of the starting-time value function $V_{i t}$ which we call temporal tolerance functions. 


\section{TEMPORAL TOLERANCE FUNCTIONS}

The optimisation problem that we consider here has the binary decision variable $x_{i \tau}$ which defines whether a project $i$ belonging to a set of candidate portfolio projects, $P$ starts at time $\tau \in\{1, \ldots T\}$. Each project $i \in P$ has a value $V_{i t}$ if it starts at time $t$, that is if $x_{i t}=1$. The cost at time $t$ for project $i$ scheduled at time $\tau$ with $t \geq \tau$ is defined to be $c_{t i \tau}$. There is an overall budget for each time period $t \in\{1,2, \ldots, T\}$ defined by $b_{t}$.

Thus in its simplest form the multiple knapsack optimisation ${ }^{1}$ problem applied to calculate the optimal portfolio is

$$
\begin{aligned}
\max _{x_{i t} \in\{0,1\}} & \sum_{i \in P} \sum_{t=1}^{T} V_{i t} x_{i t} \\
& \sum_{t=1}^{T} x_{i t} \leq 1, \forall i \in P, \\
& \sum_{i \in P} \sum_{\tau \leq t} c_{t i \tau} x_{i \tau} \leq b_{t}, \forall t \in\{1,2, \ldots, T\} .
\end{aligned}
$$

This problem has a similar structure to that found in Ghasemzadeh et al. (1999) however the authors do not mention the knapsack formalism.

Extra constraints can impose temporal dependency on a project, such as that mentioned in the introduction, or the constraint $\sum_{t=\tau}^{\tau^{\prime}} x_{i t}=0$, which prohibits projects from commencing during the time period $\left[\tau, \tau^{\prime}\right]$. Temporal dependency between projects can also be specified. For projects $i, j \in P$ then the constraint $x_{j t-\tau}=$ $x_{i t}$ specifies that these projects must start within $\tau$ time units of one another and both are either included or excluded from the optimised portfolio.

As an alternative to implementing hard constraints for the timing of the projects considered in the portfolio, one can specify a function $f_{i}:[0, T] \rightarrow \mathbb{R}^{+}$. The function $f_{i}$ is defined as the temporal tolerance (TT) function for project $i$. One sets the value function $V_{i t}=f_{i}(t)$.

The TT function specifies the allowable times a project can start, if at all, in the portfolio, and this is determined by the support of $f_{i}, \operatorname{supp}\left(f_{i}\right)$. The SME specifies a preferred scheduled time for projects $i \in P$, which is determined by $\widetilde{t_{i}}=\arg \max _{[0, T]}\left(f_{i}(t)\right)$. In this paper the tolerance for project $i \in P, \quad S_{i}$ may defined as $S=\sum_{t=1}^{T} \mathbf{1}_{f_{i}>0}(t)$, that is the width of $\operatorname{supp}\left(f_{i}\right)$. However, this is not appropriate for all TT functional forms as will be seen below. Furthermore, the TT function specifies the priority of choosing a particular time. Symmetric tolerance functions, assign equal values to the left or the right of the maximum. However the TT can be asymmetric, with more total value assigned to either the left or the right of the maximum $\tilde{t}$. For symmetric TT functions, the SME has no preference as to whether the project starts earlier or later than the preferred time $\tilde{t}$.

The simplest example of a TT function is the triangular function, which is often applied in the discipline of signal processing, Al-Tikrit \& Bansal (2006). With a peak at $\widetilde{t} \in[0, T]$, its definition is

$$
\operatorname{tri}(t)=\max \left(0, h\left(1-\frac{2}{w}(1-|t-\tilde{t}|)\right)\right) .
$$

The triangular function is shown in Figure 1. Its functional form allows for the adjustment of the tolerance level. The SME is asked specifically for the ideal start-times $\widetilde{t_{i}}$ and tolerance level $w_{i}$ for each project $i \in P$. If one sets the height of the triangle function $h=2 / w$, then the area integrates to unity. If one imposes $\sum_{t=0}^{T} f_{i}(t) \approx 1, \forall i \in P$, then all projects have equal total value assigned to them. Therefore, if one seeks to maximise value over the time horizon, a project's inclusion/exclusion in the portfolio will be determined by the specification of the ideal time, the tolerance level and the project cost structure.

The triangular function is symmetric in time, implying equal value for assigning the project to the left or the right of the ideal time $\widetilde{t}$. For symmetric TT functions the SME is ambivalent as to whether the project commences before or after the ideal time $\widetilde{t}$. One can also define an asymmetric triangle function such as that from equation 6 below. Here, the SME is asked the ideal time $\widetilde{t_{i}}$ and the tolerances before the ideal time $w_{i 1}$, and post the ideal time $w_{i 2}$.

\footnotetext{
${ }^{1}$ The multiple knapsacks are the budgets for each time period, $b_{t}$.
} 
G. Calbert, Project portfolio optimisation and scheduling using temporal tolerances
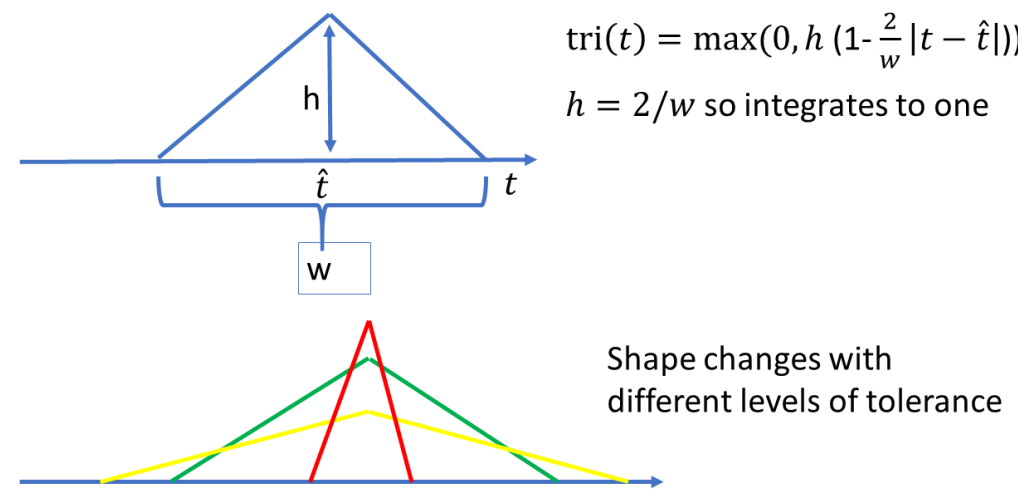

Figure 1. The triangular temporal tolerance function, noting that the area integrates to one. The tolerance level is specified by the width $w$.

In equation 6 the width on the left hand side is $w_{1}$ and on the right hand side $w_{2}$. Again the total area of this asymmetric TT function is unity when the height $h=\frac{2}{w_{1}+w_{2}}$.

$$
\text { asymtri }(t)= \begin{cases}0 & \text { if } t \leq \widetilde{t}-w_{1} \\ \frac{t-\tilde{t}}{2 \widetilde{t}-w_{1}}+h & \text { if } \widetilde{t}-w_{1}<t \leq \widetilde{t} \\ \frac{h}{w_{2}}\left(\widetilde{t}+w_{2}-t\right) & \text { if } \tilde{t} \leq t \leq \tilde{t}+w_{2} \\ 0 & \text { if } t>\widetilde{t}+w_{2}\end{cases}
$$

Other TT functions may be specified. As an example, for a project $i$, one could define the TT function ${ }^{2}$

$$
f_{i}(t)=\frac{1}{2 \sqrt{A_{i} \pi}}\left(1-\exp \left(-\frac{A_{i}}{\left(t-\widetilde{t_{i}}\right)^{2}}\right)\right)
$$

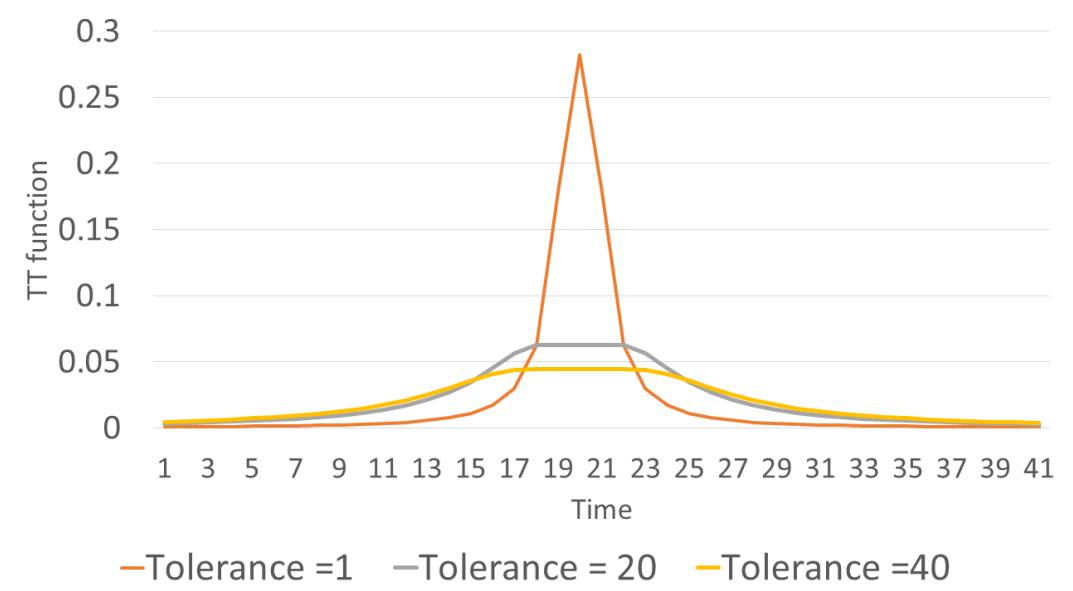

Figure 2. The shape of an alternative TT function, with the tolerance parameter being $A_{i}=1,20$ or 40 .

${ }^{2}$ The integral $\int_{-\infty}^{\infty}\left(1-\exp -\frac{1}{t^{2}}\right) d t=2 \sqrt{\pi}$ and a change of variables reveals the normalisation constant. 
The parameter $A_{i}$ is the tolerance parameter, which is not the same as the function support which is infinite. As $A_{i} \rightarrow \infty$ the width of the "flat"region, where $d^{2} f_{i}(t) / d t^{2} \approx 0$ increases. SMEs will be able to specify a tolerance interval, and the value of the tolerance parameter may be inferred from this interval. By calculating the second derivative of the TT function, one may infer that if the SME specifies a preferred time interval for project $i$ as $\left[t_{i 1}, t_{i 2}\right]$, then $A_{i}=\frac{3}{2}\left(t_{i 1}+t_{i 2}\right)^{2}$ and $\widetilde{t}=\frac{t_{i 2}+t_{i 1}}{2}$. This TT function, over different tolerance values is viewed in Figure 2

\section{OPTIMISATION EXPERIMENTS}

At this stage of the research, preliminary numerical optimisation experiments were conducted using the symmetric triangular TT function. The experiments were aimed to understanding, as tolerance levels changed for all projects uniformly, how many were admitted, the total value and the distance between the preferred commencement time and the time calculated, termed the temporal deviance, after optimisation. By definition, projects that are excluded from the portfolio, post optimisation have no defined temporal deviance.

The set of projects that were actually admitted into the portfolio is termed $P^{*} \subseteq P$. The precise definition for temporal deviance $d\left(P^{*}\right)$ is as follows. For $i \in P^{*}$ after optimisation, one measures the distance between the preferred time $\widetilde{t_{i}}$ and the optimised time $t_{i}^{*}$, normalised by the number of projects in the portfolio,

$$
d\left(P^{*}\right)=\frac{\sum_{i \in P^{*}}\left|t_{i}^{*}-\widetilde{t}_{i}\right|}{\sum_{i \in P} \sum_{t=1}^{T} x_{i t}} .
$$

In the optimisation experiments $|P|=50$ candidate projects were considered. The horizon for the timing of included projects was $T=10$. The time sequence of project costs was assumed to be invariant over the starting time, that is no inflation was assumed. Therefore, the cost of project $i$ at time $t$ which starts at time $\tau \leq t, \quad c_{t i \tau}=c_{(t-\tau+1), i, 1}$. It is assumed that $c_{t i 1} \sim U[0,100]$, that is costs per project per year are uniformly distributed between zero and one hundred cost units. It is assumed that the time dependent budget cost is modelled by $b_{t}=100+50(t-1)$. Projects in the defence domain have been analysed for their longitudinal cost structure, see Weir \& Johns (2017), however in this preliminary analysis, the focus will be on the broad results of changing TT values. A sensitivity analysis was conducted where the TT is the width of the triangular function. Here the width for all projects in $P, w$ ranges from $1 \rightarrow 20$.

The following Figure 3 graphs the optimised portfolio value $V^{*} \rightarrow 0$ as $w \rightarrow \infty$. The number of projects admitted to the portfolio $\left|P^{*}\right|$ increases as $w \rightarrow \infty$. As the TT increases the value increment of a project decreases non-linearly, noting the incremental value for a project's inclusion in the portfolio is bounded by $2 / w$. Given the number of projects included in the portfolio is bounded by fifty, the optimal value $V^{*}<50 / w$. As $w \rightarrow \infty$ then $\left|P^{*}\right|$ increases. As the TT increases, the choice of potential starting times for projects increases (has a positive reward), giving more options to include such projects whilst meeting budget bounds.
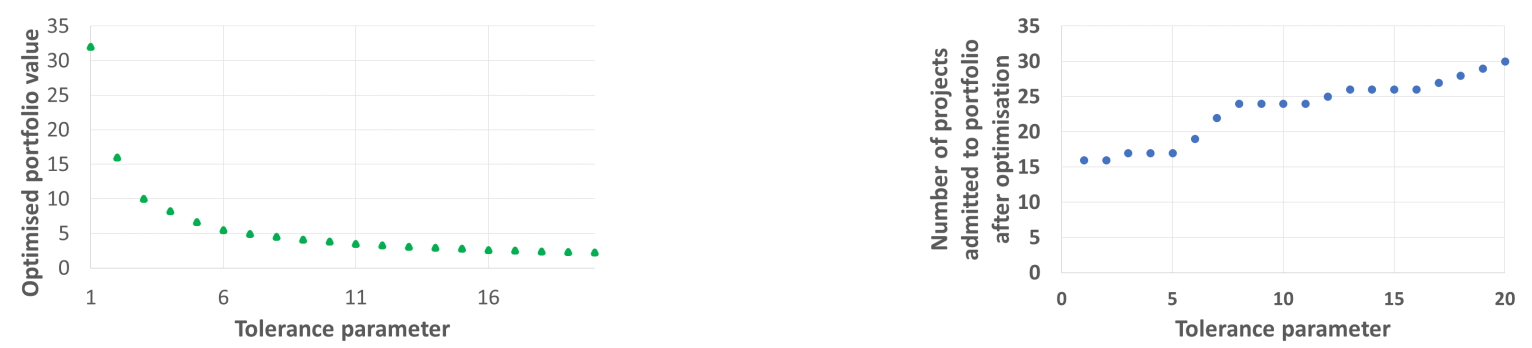

Figure 3. Optimised portfolio value, $V^{*}$ and number of included projects, $\left|P^{*}\right|$ out of a total of fifty, as a function of the temporal tolerance parameter $w$ for the symmetric triangle function.

Figure 4 plots the portfolio temporal deviance, $d\left(P^{*}\right)$ as $w$ increases. As expected as $w \rightarrow \infty$ the temporal deviance increases. Changes in project timings are met with decreased increments in reward. Therefore, as $w \rightarrow \infty$ projects have greater freedom to move to meet budget constraints. 
G. Calbert, Project portfolio optimisation and scheduling using temporal tolerances

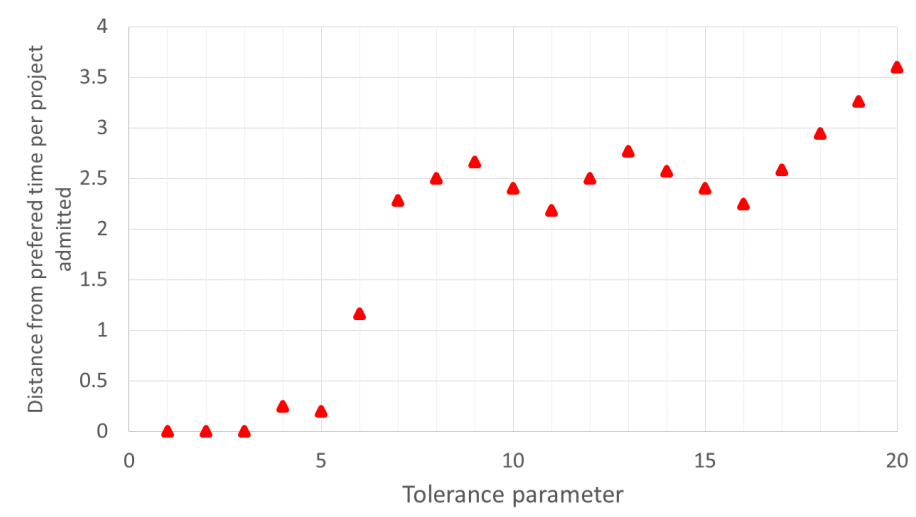

Figure 4. The temporal deviance $d\left(P^{*}\right)$ for the optimised portfolio as the temporal tolerance parameter increases.

\section{CONCLUSIONS}

In this paper, value determination was done by imposing a specific functional form, that of the TT function. By structuring and adopting an appropriate functional form for the starting-time value function, one can restructure the vaguely defined notion of project value to that of an appropriate time, and appropriate level of forbearance in the choice of timing. No doubt there are many possible forms for a temporal tolerance function and three were discussed here, two symmetric and one asymmetric.

At this stage of the research, it is unknown as to whether there is a preferred tolerance function, for multiple knapsack problems, dependent on the longitudinal structure of project cost functions and the budget. Furthermore, this work is an example of what might be larger research effort to understand the statistics or statistical mechanics of multiple knapsack problems and their dependence on the functional forms of the value function and cost structure.

Generally, starting time value functions can take on many forms. For instance value functions that are monotonically decreasing in time have the effect of rewarding projects that start earlier in time, by definition. Further research is required to understand how the portfolio structure changes with the aforementioned stating time value function form. Some initial research steps into statistical structure have been taken in multiple constraint knapsack problem, see Korutcheva et al. (1994). Such research is required to form an intuitive understanding of the optimisation results when using the knapsack approach to defence portfolio design. ${ }^{3}$

\section{ACKNOWLEDGEMENT}

Thank you Terence Weir for some brief discussions on different value function shapes. The author would like to thank two anonymous reviewers for excellent feedback on the paper.

\section{REFERENCES}

Al-Tikrit, M. \& Bansal, V. (2006), 'Theory of orthogonal triangular functions and its application', International Journal of Mathematical Education in Science and Technology . [Online; accessed 16-July2021].

Ghasemzadeh, F., Archer, N. \& Iyogun, P. (1999), 'A zero-one model for project portfolio selection and scheduling', Journal of the Operational Research Society 50, 745-755.

Harrison, K. R., Elsayed, S., Garanovich, I., Weir, T., Gallister, M., Boswell, S., Taylor, R. \& Sarker, R. (2020), Multi-Period Project Selection and Scheduling for Defence Capability-Based Planning, in '2020 IEEE International Conference on Systems, Man, and Cybernetics', IEEE, p. In Press.

Heckman, D (2001), 'Bayesian Graphical Models and Networks', Science Direct. Accessed 1 September, 2021 [Online].

URL: https://www.sciencedirect.com/science/article/pii/B0080430767004411

\footnotetext{
${ }^{3}$ In the multiple constraint knapsack problem one has a choice to include an item or not and there is more than one constraint, such as bounds on the length and breadth. In the multi-knapsack problem one chooses which particular knapsack to put an item in, with one or a number of constraints on each of the multiple knapsacks.
} 
G. Calbert, Project portfolio optimisation and scheduling using temporal tolerances

Kellerer, Hans and Pferschy, Ulrich and Pisinger, David (2004), Multidimensional knapsack problems, in H. Kellerer, ed., 'Knapsack Problems', Springer, https://www.springer.com/gp, chapter 9, pp. 235-283.

Korutcheva, E., Opper, M. \& Lopez, B. (1994), 'Statistical mechanics of the knapsack problem', Journal of Physics A: General and Mathematical 27, 645-650.

URL: https://iopscience.iop.org/article/10.1088/0305-4470/27/18/001/pdf

Levinson, A. (2009, (accessed 15 July 2021)), 'Happiness as a tool for valuing public goods', https: / / voxeu.org/article/valuing-public-goods-using-happiness-surveys.

Lumen, B. e. (2021, (accessed 6 July, 2021)), 'Public goods', https: / / courses. lumenlearning . com/boundless-economics/chapter/public-goods/.

Weir, T. \& Johns, K. (2017), Longitudinal models for project expenditure plans, in 'Proceedings of the 22nd International Congress on Modelling and Simulation.', number December, pp. 695-701.

URL: http://www.mssanz.org.au/modsim2017/D3/weir.pdf 